\title{
Adaptación de una metodología de especificaciones en la calificación y retroalimentación en la enseñanza de cursos de ciencias básicas en una carrera de la salud para mejorar los aprendizajes y la motivación
}

\author{
Adaptation of a specifications gradings and feedback methodology for basic sciences courses \\ teaching in a health science career to improving learning and motivation \\ Mauricio Cerda ${ }^{1}$, Karen Gormaz², Héctor Vega ${ }^{3}$, Víctor Castañeda ${ }^{2}$, Diego Varela ${ }^{3}$, Mariana Casas ${ }^{3}$, \\ Jocelyn Dunstan ${ }^{4}$, Milton de la Fuente ${ }^{3}$, Natasha Kunakov ${ }^{5}$
}

\section{Resumen}

Introducción: la enseñanza de cursos de ciencias básicas en carreras de la salud es un desafío por no estar directa e inmediatamente relacionada con el ámbito profesional. Por otra parte, las condiciones de estrés que ha impuesto el trabajo a distancia requiere de metodologías motivantes, y, que a su vez permitan una evaluación significativa. Objetivos: reportar las adaptaciones metodológicas y los resultados de una adaptación local de la metodología de especificaciones de las calificaciones y retroalimentación del trabajo. Métodos: se aplica una metodología de formación basada en la retroalimentación en el curso de Física para estudiantes de Tecnología Médica (N=106) durante un semestre. Las calificaciones promedio de los estudiantes fueron comparadas con las obtenidas en años anteriores. Para evaluar el desempeño docente se realizaron 2 encuestas a los estudiantes. El cumplimiento de los logros de aprendizaje se midió mediante auto-evaluación (escala likert 1 a 5) al inicio y término de cada uno de los cuatro capítulos. Resultados: las reprobaciones y eliminaciones de estudiantes en el curso fueron menores a años anteriores, siendo las notas significativamente mayores subiendo desde 4,89 a 6,29 (escala de 1 a 7, p<0,001). Los estudiantes se mostraron en un $95 \%$ satisfechos con el desempeño docente y finalmente, la auto-evaluación de logros de aprendizaje mostró un aumento promedio de 1 punto. Conclusiones: la metodología de evaluación basada en especificaciones adaptada a dos entregas y con evaluaciones en una escala no-binaria mejoró el rendimiento, los logros de los aprendizajes esperados y la motivación de los estudiantes.

Palabras clave: especificaciones; evaluación; ciencias; dominio.

\begin{abstract}
Background:Teaching basic science courses in health careers is a challenge because these courses are not directly linked to professional practice. On the other hand, the stressful conditions imposed by distance work require motivating methodologies and a meaningful evaluation. Objectives: To report the methodological adaptations and the results of a local adaptation of the specifications grading and feedback methodology. Methods: A training methodology based on feedback is applied in the Physics course for Medical Technology students $(\mathrm{N}=106)$ during one semester. We compared the students' average grades to those obtained in previous years with the same topics. To evaluate the teaching performance, we conducted two student surveys. We measure compliance with learning achievements by self-assessment (Likert scale 1 to 5 ) at the beginning and end of each of the four chapters. Results: Failures and eliminations of students in the course were lower than previous years, with significantly higher grades from 4.89 to 6.29 ( $p<0.001$ ). The students were $95 \%$ satisfied with the teaching performance, and finally, the self-evaluation of learning achievements showed an average increase of 1 point. Conclusion: The evaluation methodology based on specifications adapted to two deliveries and evaluations on a non-binary scale improved the performance, expected learning achievements, and students' motivation.
\end{abstract}

Keywords: specification; grading; sciences; learning for mastery.

Fecha de envío: 2021-10-27 - Fecha de aprobación: 2021-12-27

(1) Programa de Biología Integrativa, Instituto de Ciencias Biomédicas y Centro de Informática Médica y Telemedicina, Facultad de Medicina, Universidad de Chile.

(2) Departamento de Tecnología Médica, Facultad de Medicina, Universidad de Chile.

(3) Programa de Fisiología y Biofísica, Instituto de Ciencias Biomédicas, Facultad de Medicina, Universidad de Chile.

(4) Iniciativa de Datos e Inteligencia Artificial y Centro de Modelamiento Matemático Facultad de Ciencias Física y Matemáticas, Universidad de Chile.

(5) Departamento de Educación en Ciencias de la Salud, Facultad de Medicina, Universidad de Chile.

Autor de correspondencia: mauricio.cerda@uchile.cl

ARS MEDICA Revista de Ciencias Médicas Volumen 46 número 4 año 2021 


\section{Introducción}

En las carreras universitarias relacionadas a la salud, es necesario desarrollar saberes en el ámbito científico general y en temas específicos básicos, por ello, los primeros años tienen cursos de asignaturas tales como química, matemáticas, y física. La enseñanza de estas asignaturas es especialmente desafiante para docentes y alumnos pues no están directamente relacionadas con el ámbito profesional para el cual se están formando.

En este contexto, el curso de Física II de la carrera de tecnología médica de la Universidad de Chile, es un curso de primer año, con aproximadamente 100 estudiantes (106 en su edición 2020). Dicho curso posee 5 créditos o 135 horas semestrales con 81 directas y 54 no presenciales. Las horas directas se distribuyen en 2 bloques semanales: 1 hora 30 minutos y 3 horas. El curso está organizado en 4 capítulos: termodinámica, ondas, electromagnetismo, y óptica. Los logros esperados de aprendizaje son: resolución de problemas de física en manera individual y grupal, e Interpretar fenómenos físicos experimentales. Además, posee una actividad final, realizada en conjunto con el curso de matemáticas II que se imparte en paralelo. En el curso participa un equipo de 4 a 6 docentes, 4 ayudantes, y 1 secretaria docente.

Al finalizar la edición 2019, la encuesta de satisfacción aplicada a los estudiantes indicó una buena evaluación general, pero una desconexión entre el tipo de preguntas realizadas en las actividades presenciales y las evaluaciones generales de cada capítulo. La metodología de evaluación utilizaba preguntas de alternativas que, si bien agilizaban el proceso, no permitían una real retroalimentación, ni una evaluación del desarrollo de los logros de aprendizaje del curso en los dominios de tecnología en biomedicina, genérico transversal, e investigación. La situación sanitaria durante 2020, obligó a realizar todas las actividades de manera remota, por lo cual, como equipo docente, nos vimos conminados a buscar maneras alternativas de lograr interacción con los estudiantes. Y, paralelamente, lograr un formato de curso que fuera satisfactorio para todos los participantes, docentes y estudiantes, consciente de los reportes de bajo ánimo, y falta de concentración en estudiantes (ELSAM, 2020).

Por ello se planteó un cambio en la metodología de evaluación, adaptando los 15 criterios del Specifications Grading (Nilson, 2015), entre ellos evaluar el aprendizaje efectivo y reducir el estrés en estudiantes. Esta metodología plantea la entrega de tareas/trabajos y su evaluación con rúbricas simples (aprueba o no, según las expectativas de logro del curso, que son conocidas) y la posibilidad de que el estudiante pueda volver a entregar su trabajo corregido para una nueva evaluación tantas veces como sea necesario.
Adicionalmente, se incorporó la retroalimentación, que en el ámbito docente ha demostrado ser muy eficiente en el proceso de formar hábitos y actitudes, así como en el aprendizaje de desempeños. Esta es una técnica y se refiere a la entrega de información al estudiante acerca de su desempeño, en cuanto se acerca o aleja de los logros esperados de aprendizaje, todo esto con el propósito de mejorar este desempeño (Ende, 1987). Esta técnica requiere de 2 elementos fundamentales, primero, que el estudiante conozca cuales son los logros de aprendizaje que debe obtener durante el proceso de formación y segundo que se establezca una buena relación docente - estudiante.

Considerando lo anterior, se buscó una adaptación al contexto local: con docentes con tiempos acotados, la situación sanitaria, y la reglamentación de la Universidad de Chile. En concreto cada evaluación, incluyendo los trabajos prácticos, ya sea individual o grupal, tuvo un primer momento de evaluación formativa con una instancia de retroalimentación, y luego una evaluación final, calificada, es decir, sumativa.

El siguiente trabajo describe los cambios realizados y los resultados obtenidos en el curso de Física Il de la carrera de tecnología médica de la Universidad de Chile durante el 2020, reportando las diferencias cuantitativas y cualitativas observadas respecto de versiones anteriores, 2018 y 2019, destacando las lecciones aprendidas para la docencia de asignaturas científicas en carreras de la salud en el contexto chileno del año 2020.

\section{Metodología}

\section{Procedimiento}

En el curso Física II - 2020 fueron eliminadas todas las evaluaciones tradicionales (certámenes, evaluaciones globales de cada capítulo, examen final), y reemplazadas por desarrollar una tarea semanal. Las tareas consistían en 3 a 4 ejercicios por cada capítulo, más una actividad práctica grupal a realizar en horas no presenciales. Las indicaciones para realizar cada tareas fueron publicadas con 1 mes de anticipación en una guía de trabajo, con 2 fechas de entrega: una entrega formativa, sobre la cual se entregaba retroalimentación individual escrita y posteriormente la entrega final, que era calificada entre 1,0 y 7,0. La calificación en cada uno de los 4 capítulos fue el promedio de las calificaciones de las tareas realizadas, y para asegurar el logro de las competencias planificadas, la nota final se calculó como el promedio de los capítulos, siendo condición necesaria que cada capítulo fuera aprobado, es decir, presentara una calificación mayor o igual a 4,0.

Se realizó una encuesta voluntaria de desempeño docente intermedia (mitad de semestre) y otra al final del curso. También cada estudiante debía realizar de manera individual y voluntaria, una autoevaluación por logro de aprendizaje en cada capítulo. 
Cerda et al.

Tabla 1: Comparación de metodología docente en 2018, 2019 y propuesta en 2020.

\begin{tabular}{l|l|l}
\hline Aspecto & \multicolumn{1}{l}{ Año 2018, 2019 } & \multicolumn{2}{l}{ Año 2020} \\
\hline Modalidad Seminario & $\begin{array}{l}\text { Presencial con entrega de guía que lleva una pauta de } \\
\text { evaluación }\end{array}$ & $\begin{array}{l}\text { Virtual sincrónico con entrega de guía que lleva una pauta } \\
\text { de evaluación }\end{array}$ \\
\hline Retroalimentación & $\begin{array}{l}\text { Sólo se entrega la guía de ejercicios resuelta. Oportunidad } \\
\text { de entrega era irregular, promedio 2 semanas. }\end{array}$ & $\begin{array}{l}\text { Si, tareas revisadas por ayudante, informes revisados por } \\
\text { docentes; en ambos casos se entregaban retroalimentación } \\
\text { individual por escrito en 48 horas. }\end{array}$ \\
\hline Calificación final del curso & $\begin{array}{l}\text { Promedio de evaluaciones de desarrollo y prácticos y y } \\
\text { controles. }\end{array}$ & Promedio de calificaciones de capítulos. \\
\hline Calificación capítulo & Evaluación Prueba de desarrollo extensa & $\begin{array}{l}\text { Promedio de actividades de cada capítulo (calificaciones } \\
\text { de tareas y prácticos) }\end{array}$ \\
\hline Examen & Prueba de desarrollo extensa, con posible eximición & No \\
\hline
\end{tabular}

\section{Análisis estadístico}

Las tablas de datos se construyeron con Excel. Para realizar comparaciones de medias se utilizó un t-test o el test de Wilcoxon-Mann-Whitney dependiendo de la distribución de los datos. En todos los test se utilizó un p-valor de significancia del 5\%. Para realizar los test mencionados se utilizó el paquete estadístico $R$ (Core Team, 2021).

\section{Resultados}

Desempeño de los estudiantes (2018, 2019 y 2020)

En la figura 1 se resume la evolución de las tasas de reprobación, eliminación del curso y el promedio de calificaciones finales. Podemos mencionar que las reprobaciones disminuyeron desde un $7,8 \%$ a un $1 \%$, y que las eliminaciones, pese al duro contexto también disminuyeron respecto a 2019 desde un 4\% en 2019 a un $1 \%$ en 2020 . Las notas por otra parte aumentaron de manera sustancial y significativa desde 4,89/7,00 a 6,29/7,00 ( $p<0,001)$.
A

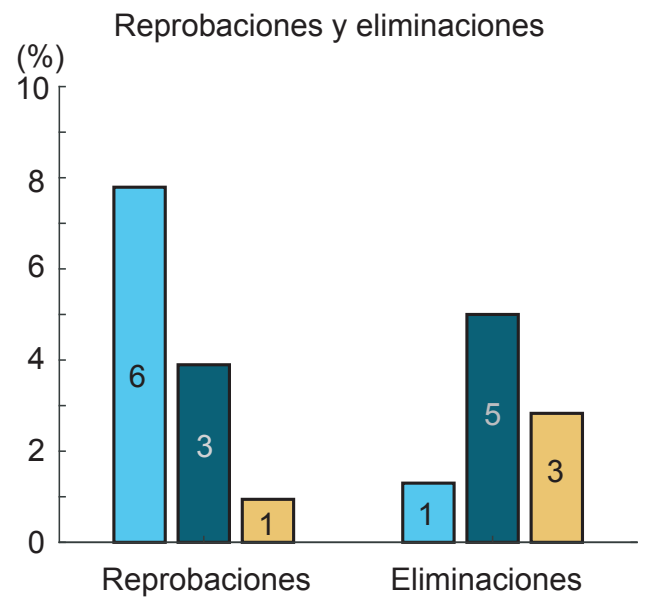

B

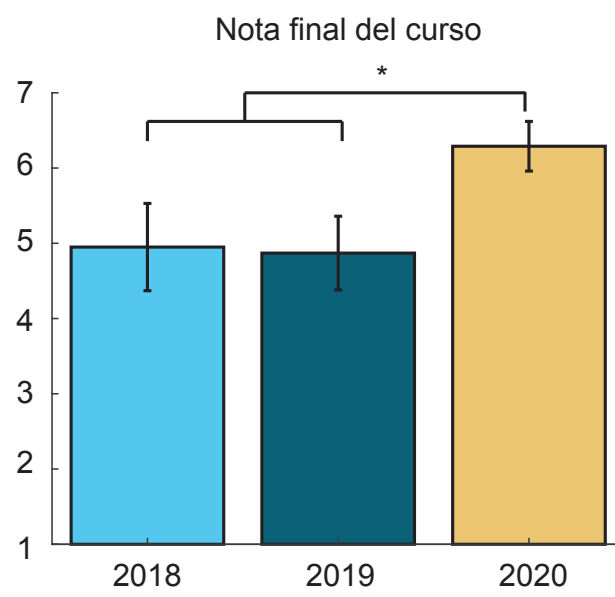

Figura 1: Desempeño de los estudiantes en el curso de Física II. Colores indican años. A Porcentajes de reprobación eliminación (números en barras indican n). B Nota final promedio final del curso.

\section{Evaluación del desempeño docente (2020)}

En el año 2020 durante el desarrollo del curso se realizaron 2 encuestas de desempeño docente, en el intermedio y hacia el final del curso. En ambas encuestas se realizaron las mismas preguntas cuyos resultados resumimos a continuación.
En la tabla 2 se muestra el resumen de los resultados. Se puede apreciar que la evaluación de desempeño es mayor en la evaluación final (95\%) respecto a la intermedia (88\%). Por otra parte, el área de evaluación más baja fue asociada a la dedicación de tiempo (80\%) en la encuesta intermedia y la más alta la Gestión del clima del aula (95\%). En la encuesta final las evaluaciones por áreas tuvieron una baja dispersión con valores entre 95\% a 97\%. 
Cerda et al.

Tabla 2: Encuestas de desempeño docente 2020.

\begin{tabular}{lll}
\hline Área & Intermedia & Final \\
& (1 a 4) & (1 a 6) \\
\hline Uso de espacios para interacción virtual (3 preguntas) & $3,5(88 \%)$ & $5,8(97 \%)$ \\
\hline Uso de metodologías participativas y apoyo al trabajo autónomo (4 preguntas) & $3,4(85 \%)$ & $5,7(95 \%)$ \\
\hline Estrategias de evaluación (4 preguntas) & $3,7(93 \%)$ & $5,7(95 \%)$ \\
\hline Gestión del clima del aula (2 preguntas) & $3,8(95 \%)$ & $5,7(95 \%)$ \\
\hline Dedicación de tiempo (1 pregunta) & $3,2(80 \%)$ & $5,8(97 \%)$ \\
\hline Promedio & $3,5(88 \%)$ & $5,7(95 \%)$ \\
\hline
\end{tabular}

En la encuesta de desempeño intermedio $(\mathrm{N}=11)$ se pidieron señalar tres aspectos positivos a mejorar y 3 aspectos por mejorar. Al analizar estos textos (texto libre) de manera cualitativa, todos los comentarios positivos mencionaron la organización (100\%) y con menor cantidad de menciones el clima en el aula (27\%), y el tipo de evaluación (18\%). Entre los aspectos a mejorar ( $\mathrm{N}=8$ ) se menciona principalmente el tiempo de dedicación (87\%) y en menor medida la organización (50\%).

En la encuesta de desempeño final se pidió indicar aspectos positivos y a mejorar directamente. Los análisis de los aspectos positivos $(\mathrm{N}=13)$ muestran que la organización (61\%) es lo más destacado, luego el clima en el aula (53\%), la metodología de evaluación (15\%), y también felicitaciones (23\%). En los aspectos a mejorar $(\mathrm{N}=2)$ se pide una profundización de un tópico (50\%), e incorporar la evaluación con una instancia sincrónica (50\%).

Adicionalmente, se comparó el desempeño de este equipo docente con los resultados promedios obtenidos por el resto de los cursos de la Facultad de Medicina (FMED) en el mismo periodo, siendo superior al menos en promedio en:

- Uso de espacios para interacción virtual (5,8 vs FMED 5,6).

- Uso de metodologías participativas y apoyo al trabajo autónomo $(5,7$ vs FMED 5,5).

- Estrategias de evaluación (5,7 vs FMED 5,4).

\section{Auto-evaluación de la metodología y cumplimiento de logros de aprendizajes (2020)}

Durante la versión 2020 del curso Física II se realizaron encuestas de auto-evaluación del aprendizaje voluntarias: la primera en el mes 1 del curso (56/106 respuestas), y luego al inicio y al final de cada capítulo. Capítulo de onda: 57 encuestas iniciales, y 19 finales. Capítulo de electromagnetismo: 14 y 7. Capítulo de óptica: 38 y 17. Dichas encuestas preguntaban a los estudiantes por aspectos metodológicos del curso y por el cumplimiento de los logros de aprendizaje específicos de cada capítulo, al iniciarlo y al terminarlo.

Sobre la metodología, se puede apreciar que en general los estudiantes valoraron positivamente la metodología realizada, ver figura 2. Existe un grado de desacuerdo en dos preguntas. La primera "El tiempo dedicado por mi, al curso, está dentro de las horas planificadas" (42/61 en acuerdo y 5/61 en desacuerdo) se puede interpretar como que la modalidad de un trabajo semanal, en un grupo reducido de estudiantes requirió de mayor tiempo semanal, o que al menos tuvieron esa percepción. La segunda pregunta con 2 estudiantes en desacuerdo "Las sesiones sincrónicas han favorecido mi aprendizaje", resulta interesante pues si bien es un porcentaje menor, se puede interpretar que la retroalimentación asincrónica pudiera reemplazar la interacción en clase con el docente.

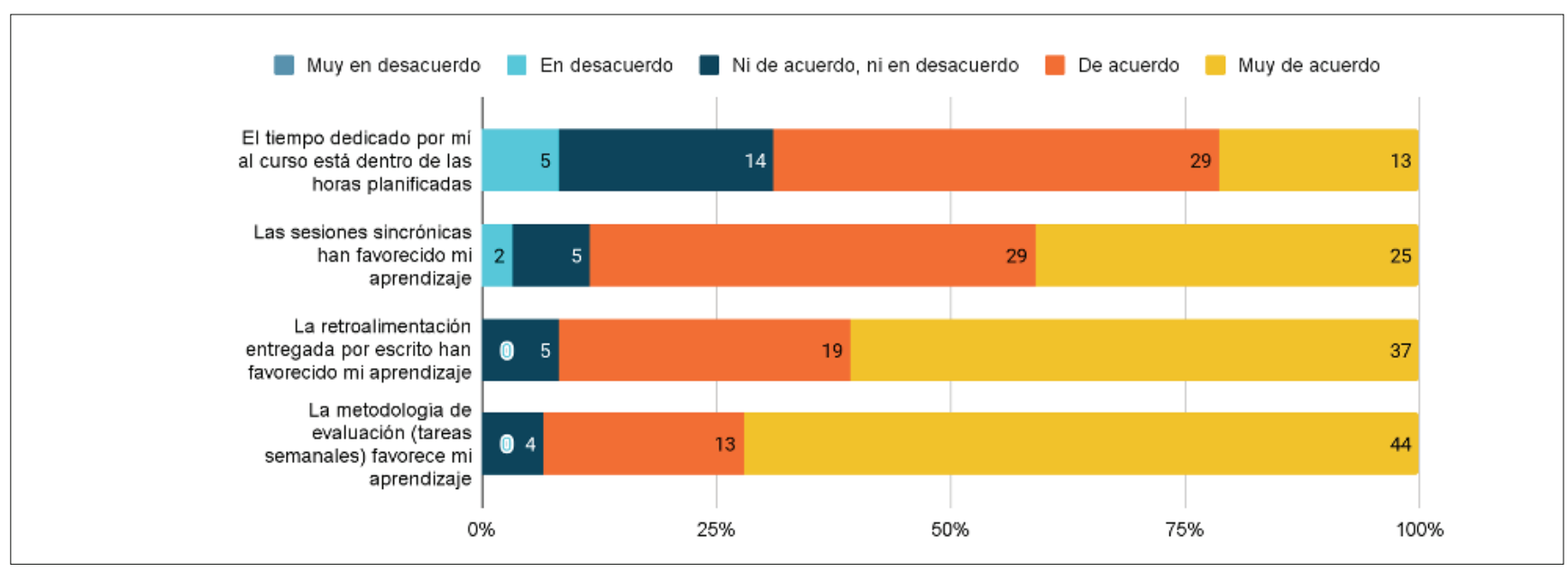

Figura 2: Valoración de la metodología aplicada en el curso. 
Se midió cuántos estudiantes indicaron haber efectivamente mejorado en alguno de los logros de aprendizaje propuesto al terminar el capítulo. Es decir, en qué medida el curso, en su conjunto, está favoreciendo su aprendizaje.

Con el fin de resumir los resultados, en este análisis tratamos la escala de valoración numéricamente como: Muy en desacuerdo $=1$, En desacuerdo = 2, Ni de acuerdo, ni en desacuerdo $=3$, De acuerdo $=4$, Muy de acuerdo $=5$. Luego, si un estudiante indica una valoración del grado de cumplimiento del logro de aprendizaje igual a 1 al inicio del capítulo, y luego igual a 4 al final del capítulo, entonces la mejora es de 3 puntos (4-1).

La autoevaluación inicial del promedio del capítulo de ondas fue de 2,98 $\pm 0,61$ y al final $4,11 \pm 0,67$ (aumento de 1,13 $\pm 0,73, N=19$ ). La autoevaluación inicial del promedio del capítulo de electromagne-

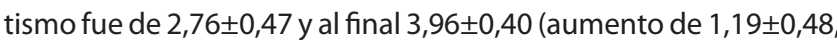
$\mathrm{N}=7$ ). La autoevaluación inicial del promedio del capítulo de óptica fue de 3,11 $\pm 0,73$ y al final 4,10 $\pm 0,76$ (aumento de $0,98 \pm 1,17, N=17$ ). Los resultados muestran que en general el curso, al menos en términos de auto-evaluación, aumenta, en promedio, en alrededor de 1 punto el nivel de logro de los resultados de aprendizaje. Para resumir el cambio entre el inicio y el término de cada capítulo la Figura 3 ilustra la valoración promedio por estudiante y por capítulo.

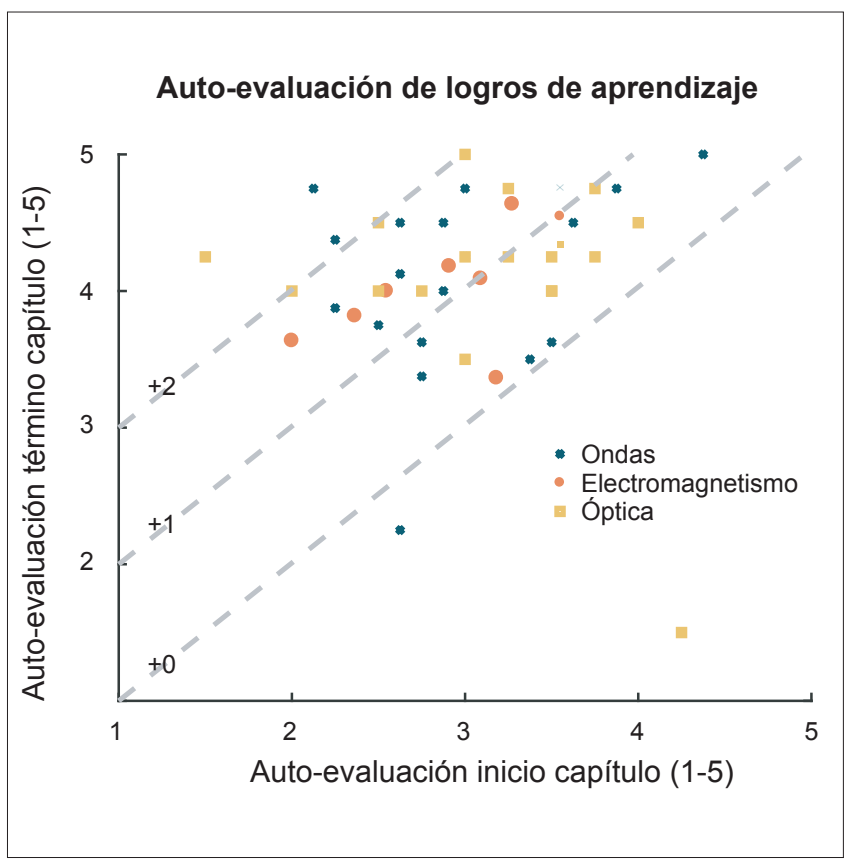

Figura 3: Auto-evaluación de los logros de aprendizaje individual por capítulo. Cada punto representa la auto-evaluación de un estudiante. Las líneas discontinuas son referencia cuando no hay mejoras $(+0)$, mejoras de 1 punto $(+1)$ y de 2 puntos $(+2)$.

\section{Otros aspectos}

Cabe señalar que el equipo docente en 2020 estaba compuesto por 6 académicos de diferente formación inicial (físicos, informáticos, bioquímicos), y que conforman una unidad docente con 5 años de experiencia. Esta experiencia interna permitió la flexibilidad para innovar, y dio pie a conversaciones abiertas sobre las inquietudes iniciales. La principal de estas inquietudes fue la dedicación horaria que la metodología realizada requería.

En el año 2019 un docente dedicó, en promedio, 5 horas semanales, divididas en 3 horas de docencia directa y 2 horas de planificación (reuniones, preparar material). En el año 2020 estimamos que ese volumen horario aumentó en al menos 1 hora (1/5 o 20\%). Esto se logró cubrir principalmente gracias a la colaboración de cuatro ayudantes, que realizan la retroalimentación escrita de cada actividad. Sin embargo, el encargado de curso requirió de un tiempo adicional para coordinar a los ayudantes y manejar correcciones de mayor dificultad, que se estimó en 4 horas semanales (2/5 o 40\%). Este tiempo adicional fue requerido para asegurar la calidad de las retroalimentaciones y su entrega oportuna.

La metodología propuesta se basa en la confianza en los estudiantes. Si bien se detectaron casos de copias aisladas en las tareas semanales (10 de aproximadamente 2200 entregas o $0,45 \%$ de tareas en el semestre), estos fueron sancionados según la reglamentación y se citó a los estudiantes de manera personalizada y sincrónica. Sin embargo, queda la inquietud de hasta qué punto las tareas se realizaban de manera individual y no de manera colectiva.

En un curso como Física II, se busca desarrollar habilidades científicas en los estudiantes, y realizar experimentos simples y reportarlos de manera clara, es una actividad fundamental en esta línea. Por esto se decidió hacer actividades prácticas en el hogar como parte de cada capítulo:

- Termodinámica. Estudio computacional (usando R) sobre probabilidades y conceptos de micro y macro estado (LoPresto, 2010).

- Ondas. Estudio de la velocidad de propagación de una onda mecánica en una cuerda mediante la cámara de un teléfono.

- Electromagnetismo. Aislamiento electromagnético de un teléfono celular.

- Óptica. Construcción de lentes convexos de fabricación casera. En la Figura 4 se muestra un grupo de estudiantes realizando un práctico en su hogar. 


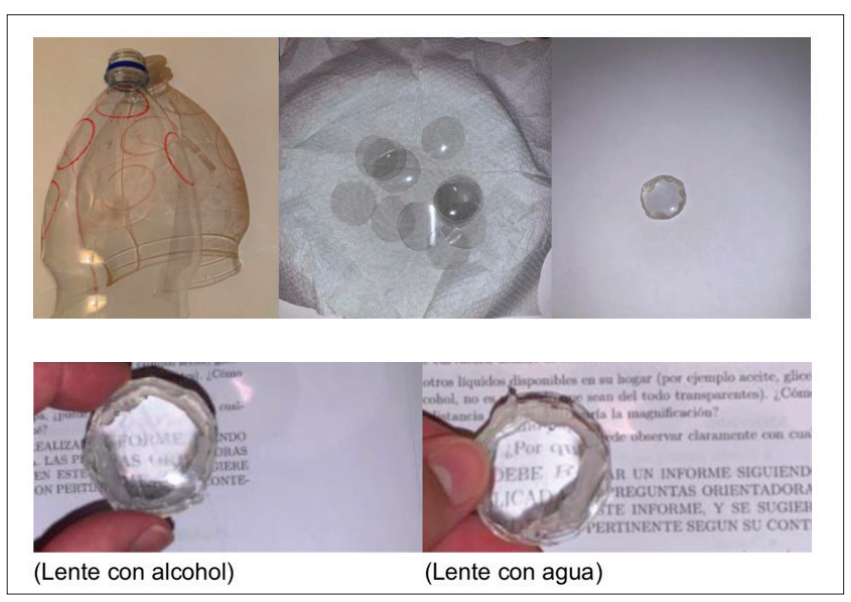

Figura 4: Pasos de una actividad práctica en el hogar. En la figura se muestra la construcción de un lente casero en base a una botella plástica, con estos lentes se exploró el aumento (distancia focal) que generan medios con diferente índice de refracción.

Los prácticos y sus informes requieren de experiencia científica, por lo que el equipo decidió que fueran los académicos quienes realizaran la retroalimentación y evaluación, siguiendo la misma dinámica de las tareas semanales realizadas por los ayudantes. Para el equipo docente fue muy gratificante ver la mejora en la calidad de los informes, retroalimentar a los estudiantes y la posterior entrega de versiones corregidas de sus informes. Muestra de ello son las menciones cualitativas en las encuestas intermedias y final del curso (2/11 y $3 / 13$ respectivamente).

\section{Discusión}

\section{Adaptación metodológica}

La metodología basada en especificaciones y que motiva este trabajo (Nilson, 2015), ya ha sido aplicada satisfactoriamente en dominios del saber tales como: computación (Quintana \& Quintana, 2020), veterinaria (Hughes et al., 2020), y nutrición (Pope et al., 2020) como una alternativa metodológica resiliente al trabajo remoto en 2020. La implementación reportada por dichos autores es identificar tareas simples (por ejemplo, asistir a clases, hacer lecturas, entregar ejercicios, entregar informes) y evaluar en una escala binaria (pasa/reprueba) en donde se puede re-enviar cada actividad. La experiencia reportada en este artículo es una adaptación de la metodología de especificaciones, con un número limitado de entregas (2) por actividad y donde las evaluaciones son en una escala (1 a 7). Es decir, es una opción intermedia que permite planificar tanto el volumen como la temporalidad del trabajo realizado, y también entrega evaluaciones en una escala familiar para los estudiantes. Es importante destacar, que ya con la retroalimentación entregada, gran parte de los estudiantes alcanzaban una nota de 7 o muy cercana, lo que explica las notas significativamente mayores del curso. En cuanto al desempeño docente, los estudiantes valoraron muy positivamente la metodología, incluso mejor que otros cursos que son más cercanos al trabajo profesional en una FMED. Se debe recalcar que la adaptación genera trabajo adicional al equipo docente tanto en planificación como ejecución, que debe ser cuidadosamente planificado y comunicado. Tomado en contexto, la adaptación de metodología propuesta es una manera de transitar desde los esquemas tradicionales hacia la metodología de especificaciones y que permite, mediante la práctica, familiarizar y promover el esquema entre los equipos docentes y los estudiantes.

\section{Otros aspectos y limitaciones}

Se ha reportado en la literatura (Berry et al., 2006) que a nivel universitario el nivel de plagio, en todos sus niveles puede llegar a ser de un $90 \%$. Si bien observamos en el curso de Física Il plagio en algunas tareas, esto es claramente inferior (0.45\%). Sin embargo, las copias detectadas son copias idénticas y no otras formas parciales de plagio. Futuras adaptaciones deben incorporar esta inquietud del equipo docente, desincentivando el plagio, como el mismo Specifications Grading sugiere (Nilson, 2015).

Efectivamente la metodología aplicada muestra mejores resultados que la aplicada en años anteriores, sin embargo, requiere de una mayor dedicación docente. El apoyo brindado por los ayudantes en revisar y dar retroalimentación para las tareas semanales fue parte importante del éxito del curso, de otro modo, el equipo docente se hubiera visto sobrecargado con los plazos.

La técnica de retroalimentación, es manejada superficialmente por los docentes/ayudantes, se requiere de una habilitación formal, y estar usándola constantemente para lograr eficiencia en su aplicación.

Una limitación importante del trabajo es que en ediciones anteriores del curso no se aplicaron las mismas encuestas, lo que dificulta la comparación.

En conclusión, parece relevante que los equipos docentes cohesionados y afiatados pueden lograr soluciones exitosas en tiempos difíciles, como ocurrió con el curso de Física II de la carrera de tecnología médica de la Universidad de Chile durante esta situación sanitaria. Una metodología de formación basada en experiencias externas, puede ser adaptada exitosamente. En este caso, la metodología fue organizar las evaluaciones con dos entregas secuenciales, la primera de ellas con retroalimentación y con evaluaciones en una escala no-binaria, lo cual mejoró el rendimiento general de los estudiantes en un curso de base científica. Esta propuesta permite alcanzar altos niveles de satisfacción de los estudiantes y equipos docentes y una buena auto-evaluación de los logros de aprendizaje. 
Cerda et al.

Por ello, el equipo docente cree que el cambio metodológico puede ser mantenido a futuro. Queda por comprobar si los aprendizajes logrados se mantienen en el tiempo.

\section{Reconocimientos}

Agradecemos a todos nuestros estudiantes y ayudantes que participaron y motivaron este trabajo. Todos los autores declaran no tener conflicto de interés en este proyecto.

\section{Aspectos éticos}

Se respetaron los principios éticos propuestos por Ezekiel J. Emanuel (Emanuel et al., 2000). La información utilizada en este artículo se manejó de manera totalmente anónima. El análisis fue retrospectivo, por lo que en cada año se aplicó la mejor metodología que el equipo docente podía utilizar para la formación de los profesionales.

\section{Referencias}

Berry P, Thornton B. \& Baker R. (2006). Demographics of digital cheating: Who cheats, and what we can do about it. En M. Proceedings of the ninth annual conference of the Southern Association for Information Systems. Davis College of Business.

ELSAM. (2020). Accedido en: https://www.uchile.cl/noticias/176250/ el-golpe-de-la-pandemia-en-la-salud-mental-de-las-y-los-estudiantes 8 de octubre de 2021
Emanuel E, Wendler D. \& Grady C. (2000). What Makes Clinical Research Ethical?. Journal of the American Medical Association 283, 2701-11.

Ende J. (1983). Feedback in clinical medical education. Journal of the American Medical Association 250, 777-81.

Hughes M, Bertram SM. \& Young AM. (2020). Teaching animal behavior online: A primer for the pandemic and beyond. Ethology $127,14-31$.

LoPresto MC. (2010). A Simple Statistical Thermodynamics Experiment. Physics Teaching 48, 183-185.

Nilson LB. (2015). Specifications Grading: Restoring Rigor, Motivating Students, and Saving Faculty Time (1 a edición). Stylus Publishing, United States of America.

Pope L, Parker HB \& Ultsch S. (2020). Assessment of Specifications Grading in an Undergraduate Dietetics Course, Journal of Nutrition Education and Behavior 52, 439-446.

Quintana R. \& Quintana C. (2020). When classroom interactions have to go online: the move to specifications grading in a project-based design course, Information and Learning Sciences 121, 525-532.

R Core Team. (2021). R: A language and environment for statistical computing. R Foundation for Statistical Computing, Vienna, Austria. 